\title{
Synthesis of new stable carbenes from the corresponding 1,3- dialkylimidazolium and benzimidazolium salts
}

\author{
O.V. Starikova,* G.V. Dolgushin, L.I. Larina, T.N. Komarova, V.A. Lopyrev \\ Irkutsk Institute of Chemistry, Siberian Branch, Russian Academy of Sciences \\ 1, Favorsky str., 664033, Irkutsk, Russia \\ E-mail: star@irioch.irk.ru
}

\section{Dedicated to Boris A. Trofimov on the occasion of his $65^{\text {th }}$ birthday, with heartiest best wishes}

(received 29 May 03; accepted 03 Aug 03; published on the web 05 Aug 03)

\begin{abstract}
N-Mono-alkylated imidazoles and benzimidazoles were prepared both by the traditional method and by microwave-assisted reaction. Their dialkylated salts were obtained, which are precursors for carbenes and novel 1,3-dialkylated imidazol-2-ilidenes and -benzimidazol-2-ilidenes.
\end{abstract}

Keywords: $N$-Alkylimidazole, $N$-alkylbenzimidazole, 1,3-dialkylimidazolium halide, 1,3dialkyl-benzimidazolium halide, carbenes

\section{Introduction}

Carbenes are compounds of divalent carbon, with a lone electron pair. Since 1991, when the first stable carbene was prepared and described, ${ }^{1}$ the chemistry of carbenes has been developed greatly. Organometallic carbene complexes are of great importance as intermediates in the homogenous catalysis of such reactions as alkene rearrangements, the Heck and Suzuki couplings, alkene and alkyne polymerization, and cyclopropane formation. ${ }^{2}$ Until now, nitrogencontaining heterocyclic carbenes have been used only as addends to phosphinic ligands. At present, the great interest in nitrogen- containing heterocyclic carbenes is based on the advantages they possess as ligands for organometallic catalysts. ${ }^{3}$ They are often less susceptible to air and moisture, and are more effective than complexes of the corresponding phosphines. The aim of this work is to obtain new carbenes as prospective ligands for the design of organometallic complexes.

\section{Results and Discussion}


To date, there are several approaches to stable nitrogen-containing heterocyclic carbenes. Liquidammonia-based synthesis affords carbenes in the highest yields. ${ }^{4,5}$ Carbenes with rather bulky and branched alkyl substituents can be synthesized by cyclization reactions. ${ }^{6,7}$ Deprotonation of 1,3-dialkylated imidazolium- and benzimidazolium salts ${ }^{1,8}$ is the most common and available method for the preparation of stable nitrogen- containing heterocyclic carbenes. We have used the last method in our experimental work, trying to improve the procedure for the synthesis of 1,3-dialkylated imidazolium and benzimidazolium salts. The preparation of the salts was performed in two steps (Scheme 1).

It has been reported ${ }^{9}$ that treatment of imidazole with two equivalents of acrylic acid, either in methanolic or in aqueous medium, leads immediately to 1,3-bis-(2-carboxyethyl)imidazolium betaine However, our attempt to treat imidazole or benzimidazole with two equivalents of alkylating agent resulted in a hardly separable mixture of monosubstituted azole, quaternary dialkylated azole salt, and potassium halide. To overcome this drawback we used the quaternization of the previously prepared $N$-substituted azole by treating it with a second equivalent of alkyl halide.

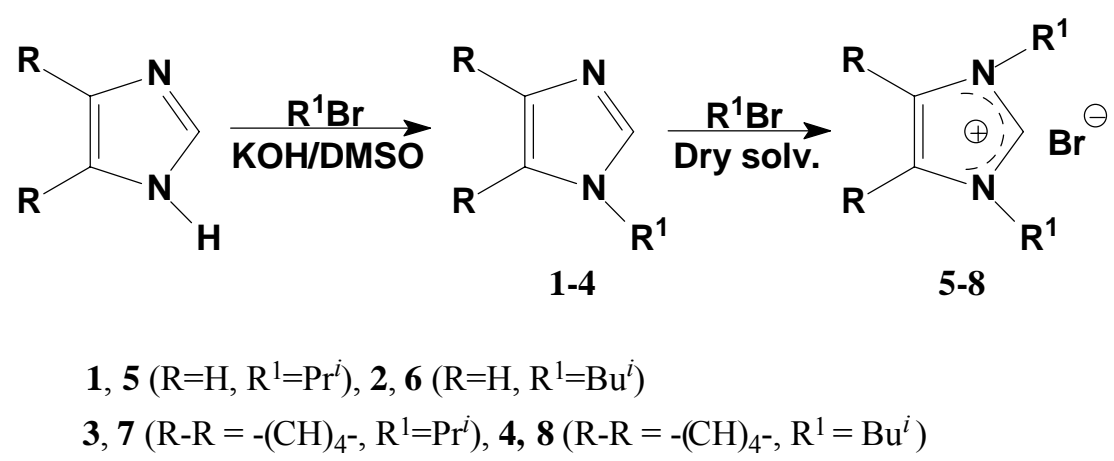

\section{Scheme 1}

We carried out the azole monoalkylation (the first stage) by the traditional method. ${ }^{10}$ Imidazole monoalkylation proceeds in $80 \%$ yield (for comparison, the yield of 1-isopropylimidazole 1 achieved in previous work ${ }^{11}$ was only $63 \%$ ), while the yield of monoalkylated benzimidazole is $40 \%$ under the same conditions. Using microwave-assisted azole alkylation (shown to be effective earlier ${ }^{12}$ ) and varying the solvent and the MW power, we obtained an increase in the yield of monoalkylated benzimidazole up to $70 \%$ (Table 1), and a reduction in the reaction time to $1 \mathrm{~h}$ (half that of the traditional method).

The highest yields were achieved using DMF as solvent, whereas increasing the reaction time from 15 to 60 min resulted in an increase in the yield of target product from 39 to $70 \%$. Solvents such as acetonitrile, methanol, or DMSO did not lead to a sufficient increase in the yield of 4.

The addition of the alkyl halide molecule to monoalkylated imidazoles and benzimidazoles, to give quaternary imidazolium- and benzimidazolium salts (the second step), was performed in anhydrous solvents, because the salts obtained are very hygroscopic (Scheme 1). The reaction proceeds readily to form N,N'-bis- adducts in good yield (Table 2). 
Table 1. Microwave assisted benzimidazole monoalkylation with iso-butyl bromide

\begin{tabular}{cccc}
\hline Solvent & $\begin{array}{c}\text { Power/ } \\
\text { W }\end{array}$ & $\begin{array}{c}\text { Time/ } \\
\text { min. }\end{array}$ & $\begin{array}{c}\text { Yield/ } \\
\%\end{array}$ \\
\hline $\mathrm{DMF}$ & 90 & 15 & 39 \\
$\mathrm{DMF}$ & 90 & 30 & 60 \\
$\mathrm{DMF}$ & 90 & 60 & 70 \\
$\mathrm{CH}_{3} \mathrm{CN}$ & 90 & 10 & $<5$ \\
$\mathrm{CH}_{3} \mathrm{OH}$ & 130 & 30 & 20 \\
& 130 & 4 & 45 \\
$\mathrm{DMSO}$ & 90 & 4 & 40 \\
\hline
\end{tabular}

Table 2. Synthesis of 1,3-dialkylated imidazolium and benzimidazolium salts

\begin{tabular}{llll}
\hline Solvent & Product & Time/ h & $\begin{array}{l}\text { Yield/ } \\
\%\end{array}$ \\
\hline Toluene & 5 & 12 & 60 \\
Propanol & 6 & 18 & 65 \\
Toluene & 7 & 26 & 35 \\
Acetonitrile & 8 & 18 & 29 \\
\hline
\end{tabular}

The new stable carbenes (symmetrical 1,3-dialkylated imidazole-2-ilidenes) 9-12 were prepared by deprotonation of the imidazolium and benzimidazolium cations of $\mathbf{5}-\mathbf{8}$ with sodium hydride under dry argon in the presence of catalytic amounts of potassium tert-butoxide (Scheme 2), according to procedures described earlier. ${ }^{1,8,13}$

Strong evidence for the formation of carbenes 9-12 is the presence of signals in their NMR spectra in the 202-226 ppm range belonging to C-2 atoms. Carbenes prepared in other work ${ }^{1,8,13}$ had shifts for C-2 in the 210-219 ppm range

\section{Conclusions}

As a result of our work, new stable carbenes have been synthesized. The effective synthesis of their precursors was accomplished using two-step alkylation of imidazoles and benzimidazoles. For the first step (monoalkylation) traditional and MW- assisted reactions were employed. The target products are of interest for the design of metal complex catalysts. ${ }^{1,3,14}$ 


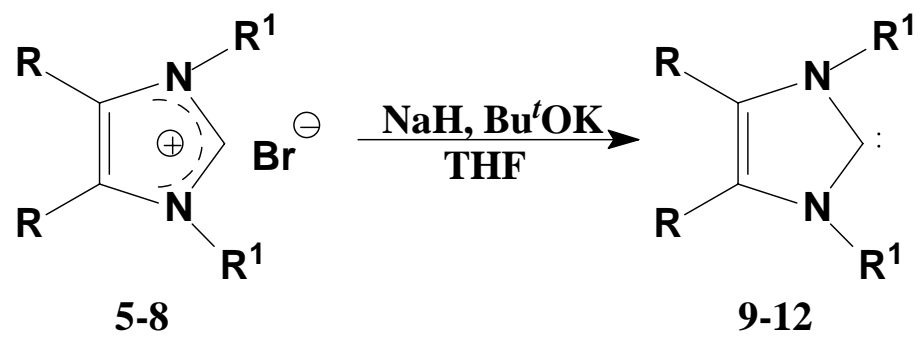

5, $9\left(\mathrm{R}=\mathrm{H}, \mathrm{R}^{1}=\mathrm{Pr}^{i}\right), \mathbf{6 , 1 0}\left(\mathrm{R}=\mathrm{H}, \mathrm{R}^{1}=\mathrm{Bu}^{i}\right)$

7, $11\left(\mathrm{R}-\mathrm{R}=-(\mathrm{CH})_{4^{-}}, \mathrm{R}^{1}=\mathrm{Pr}^{i}\right), \mathbf{8}, 12\left(\mathrm{R}-\mathrm{R}=-(\mathrm{CH})_{4^{-}}, \mathrm{R}^{1}=\mathrm{Bu}^{i}\right)$

Scheme 2

\section{Experimental Section}

General Procedures. The ${ }^{1} \mathrm{H}$ - and ${ }^{13} \mathrm{C}$ - NMR spectra were recorded on a Bruker-DPX-400 instrument $\left(400.13 \mathrm{MHz}\right.$ for ${ }^{1} \mathrm{H}, 100.61 \mathrm{MHz}$ for ${ }^{13} \mathrm{C}$ ) in $\mathrm{DMSO}-d_{6}$ solvent. ${ }^{1} \mathrm{H}$ - and ${ }^{13} \mathrm{C}$ - NMR chemical shifts were measured relative to TMS. The precision of $\delta$ - ${ }^{1} \mathrm{H}$ and $\delta-{ }^{13} \mathrm{C}$ values is 0.01 and $0.02 \mathrm{ppm}(0.1 \mathrm{~Hz}$ for cross-coupling constants). The assignments of phenylene proton signals in 3 and $\mathbf{4}$ were made using the 2D CH-CORR method: there were cross-peaks of phenylene $\mathrm{H}-7$ with $\mathrm{CH}-$ (3) or $\mathrm{CH}_{2}-$ (4) groups.

\section{General procedures for preparation of $\mathbf{N}$-mono-alkylated azoles}

Method (a). $\mathrm{KOH}(0.84 \mathrm{~g}, 0.015 \mathrm{mmol})$ was added to the solution of the azole $(0.01 \mathrm{~mol})$ in DMSO $(20 \mathrm{ml})$ and stirred at room temperature for $5 \mathrm{~h}$. Freshly distilled alkyl halide $(0.01 \mathrm{~mol})$ was added dropwise to the mixture under vigorous stirring and water-bath cooling. After $2 \mathrm{~h}$ the mixture was diluted with distilled water $(40 \mathrm{ml})$ and extracted with chloroform $(6 \times 10 \mathrm{ml})$, the organic layers were washed with distilled water to $\mathrm{pH} 7$, and dried over $\mathrm{MgSO}_{4}$. After removal of chloroform the residue was distilled under vacuum if liquid, or recrystallized from hexane.

Method (b). Reaction in microwave oven. The azole (0.01 mol), KOH (0.84 g, $0.015 \mathrm{~mol})$, DMF $(20 \mathrm{ml})$ and alkyl halide $(0.01 \mathrm{~mol})$ were placed in a flask. After the reaction in the microwave oven (parameters in Table 1) the flask was cooled. The mixture was diluted with distilled water, extracted with chloroform, then the organic layers were washed with distilled water to $\mathrm{pH} 7$ and dried over $\mathrm{MgSO}_{4}$. After removal of chloroform the residue was distilled under vacuum, if liquid, or recrystallized from hexane.

1-iso-Propylimidazole (1, $\left.\mathbf{C}_{6} \mathbf{H}_{10} \mathbf{N}_{2}\right)$. A colorless viscous liquid, prepared according to method a. b.p. $68-72^{\circ} \mathrm{C} / 3 \mathrm{mmHg}$. Found: $\mathrm{C} 65.64 ; \mathrm{H} 9.09 ; \mathrm{N} 25.27$. Calculated: $\mathrm{C} 65.42 ; \mathrm{H} \mathrm{9.15;} \mathrm{N}$ $25.43 \%$. Yield 80\%. NMR data: $\delta_{\mathrm{H}} 7.67\left(\mathrm{H}-2, \mathrm{dd},{ }^{4} J=1.3 \mathrm{~Hz},{ }^{4} J=1.1 \mathrm{~Hz}\right), 7.15\left(\mathrm{H}-4, \mathrm{dd},{ }^{3} J=1.5\right.$ $\left.\mathrm{Hz},{ }^{4} J=1.3\right), 6.95\left(\mathrm{H}-5, \mathrm{dd},{ }^{3} J=1.5 \mathrm{~Hz},{ }^{4} J=1.1\right), 4.33\left(\mathrm{CH}, \mathrm{m},{ }^{3} J=6.6\right), 1.31\left(\mathrm{CH}_{3}, \mathrm{~d},{ }^{3} J=6.6\right.$ Hz). $\delta_{\mathrm{C}} 135.35(\mathrm{C}-2), 128.43(\mathrm{C}-5), 116.79(\mathrm{C}-4), 48.35(\mathrm{CH}), 23.23\left(\mathrm{CH}_{3}\right)$.

1-iso-Butylimidazole (2, $\left.\mathbf{C}_{7} \mathbf{H}_{12} \mathbf{N}_{2}\right)$. A colorless viscous liquid, prepared according to method a. b.p. $78^{\circ} \mathrm{C} / 3 \mathrm{~mm} \mathrm{Hg}$. Found: C 67.67; H 9.81; N 22.52. Calculated: C 67.70; H 9.74; N 22.56\%. Yield 60\%. NMR data: $\delta_{\mathrm{H}} 7.59\left(\mathrm{H}-2, \mathrm{dd},{ }^{4} J=1.3 \mathrm{~Hz},{ }^{4} J=1.1 \mathrm{~Hz}\right), 7.1\left(\mathrm{H}-4, \mathrm{dd},{ }^{3} J=1.2 \mathrm{~Hz}\right), 6.9$ 
$\left(\mathrm{H}-5, \mathrm{dd},{ }^{3} J=1.2 \mathrm{~Hz}\right), 3.74\left(\mathrm{CH}_{2}, \mathrm{~d},{ }^{3} J=7.1\right) 1.95\left(\mathrm{CH}, \mathrm{m},{ }^{3} J=6.6\right), 0.8\left(\mathrm{CH}_{3}, \mathrm{~d},{ }^{3} J=6.6 \mathrm{~Hz}\right) . \delta_{\mathrm{C}}$ $137.41(\mathrm{C}-2), 128.14(\mathrm{C}-5), 119.43(\mathrm{C}-4), 53.19\left(\mathrm{CH}_{2}\right), 29.36(\mathrm{CH}), 19.34\left(\mathrm{CH}_{3}\right)$.

1-iso-Propylbenzimidazole $\left(3, \mathbf{C}_{10} \mathbf{H}_{12} \mathbf{N}_{2}\right)$. Colorless viscous liquid, prepared according to method a. b.p. $135-140^{\circ} \mathrm{C} / 1 \mathrm{~mm} \mathrm{Hg}$. Found: C 75.03; H 7.62; N 17.44. Calculated: C 74.97; H 7.55; N 17.48\%. Yield 78.7 \%. NMR data: $\delta_{\mathrm{H}} 8.39(\mathrm{H}-2), 7.85(\mathrm{H}-4), 7.26(\mathrm{H}-5), 7.28(\mathrm{H}-6)$, $7.55(\mathrm{H}-7), 4.6(\mathrm{CH}), 1.47\left(\mathrm{CH}_{3}\right) . \delta_{\mathrm{C}} 144.06(\mathrm{C}-2), 141.4(\mathrm{C}-9), 133.3(\mathrm{C}-8), 122.15(\mathrm{C}-6), 121.48$ (C-5), $119.76(\mathrm{C}-4), 110.66(\mathrm{C}-7), 47.2(\mathrm{CH}), 22.1\left(\mathrm{CH}_{3}\right)$.

1-iso-Butylbenzimidazole $\left(4, \mathrm{C}_{11} \mathbf{H}_{14} \mathbf{N}_{2}\right)$. Colorless viscous liquid, prepared according to methods a and b; b.p. $155-165^{\circ} \mathrm{C} / 1 \mathrm{Hg} \mathrm{mm}$. The compound was crystallized at room temperature; white crystals. M.p. $49-50^{\circ} \mathrm{C}$. Found: C 75.73; H 8.06; N 16.21. Calculated: C 75.82; H 8.10; N 16.08\%. The yields were $29 \%$ and $67 \%$, respectively, from methods a and $\mathbf{b}$. NMR data: $\delta_{\mathrm{H}} 8.19(\mathrm{H}-2), 7.64(\mathrm{H}-4), 7.18(\mathrm{H}-5), 7.23(\mathrm{H}-6), 7.58(\mathrm{H}-7), 4.05\left(\mathrm{CH}_{2}\right), 2.14(\mathrm{CH})$, $1.47\left(\mathrm{CH}_{3}\right) . \delta_{\mathrm{C}} 144.31(\mathrm{C}-2), 143.33(\mathrm{C}-9), 134.07(\mathrm{C}-8), 122.12(\mathrm{C}-6), 121.26(\mathrm{C}-5), 119.35(\mathrm{C}-$ 4), $110.5(\mathrm{C}-7), 51.2\left(\mathrm{CH}_{2}\right), 29.1(\mathrm{CH}), 20.2\left(\mathrm{CH}_{3}\right)$.

General procedure for preparation of 1,3-disubstituted imidazolium and benzimidazolium salts

The solvents were purified using the methods described. ${ }^{12}$ The $N$-monosubstituted azole $(0.1$ $\mathrm{mmol})$ and dry solvent $(20 \mathrm{ml})$ were placed in a two-necked flask and stirred until homogenous, then alkyl halide $(0.3 \mathrm{mmol})$ was added dropwise under stirring. After addition of all the alkyl halide, the mixture was stirred under heating for the period indicated in Table 3, the solvent was removed, and the residue washed with dry THF and dried under vacuum.

1,3-di-iso-Propylimidazolium bromide (5, $\mathbf{C}_{9} \mathbf{H}_{17} \mathbf{N}_{2} \mathbf{B r}$ ). White powder, m.p. $136-138^{\circ} \mathrm{C}$ (from THF). Found: C 46.55; H 7.18; N 12.02; Br 34.25. Calculated: C 46.36; H 7.35; N 12.01; Br 34.27\%. Yield 60\%. NMR data: $\delta_{\mathrm{H}} 9.35\left(\mathrm{H}-2, \mathrm{t},{ }^{4} \mathrm{~J}=1.7 \mathrm{~Hz}\right), 7.94\left(\mathrm{H}-4, \mathrm{~d},{ }^{4} J=1.6\right), 7.94(\mathrm{H}-5, \mathrm{~d}$, $\left.{ }^{4} J=1.6\right), 4.63(\mathrm{CH}), 1.49\left(\mathrm{CH}_{3}\right) . \delta_{\mathrm{C}} 133.65(\mathrm{C}-2), 120.71(\mathrm{C}-4), 120.71(\mathrm{C}-5), 52.3(\mathrm{CH}), 22.4$ $\left(\mathrm{CH}_{3}\right)$.

1,3-di-iso-Butylimidazolium bromide (6, $\mathbf{C}_{11} \mathbf{H}_{21} \mathbf{N}_{2} \mathbf{B r}$ ). White powder, m.p 84-86 ${ }^{\circ} \mathrm{C}$ (from THF). Found: C 51.02; H 8.10; N 11.05; Br 29.83. Calculated: C 50.58; H 8.10; N 10.72; Br $30.59 \%$. Yield 39\%. NMR data: $\delta_{\mathrm{H}} 9.27(\mathrm{H}-2, \mathrm{~s}), 7.82(\mathrm{H}-4, \mathrm{~d}, J=1.6 \mathrm{~Hz}), 7.82(\mathrm{H}-5, \mathrm{~d}, J=1.6$ $\mathrm{Hz}), 4.04\left(\mathrm{CH}_{2}\right) 2.12(\mathrm{CH}), 0.85\left(\mathrm{CH}_{3}\right) . \delta_{\mathrm{C}} 136.47(\mathrm{C}-2), 122.96(\mathrm{C}-4), 122.96(\mathrm{C}-5), 55.63$ $\left(\mathrm{CH}_{2}\right), 28.81(\mathrm{CH}), 19.16\left(\mathrm{CH}_{3}\right)$.

1,3-di-iso-Propylbenzimidazolium bromide (7, $\left.\mathbf{C}_{13} \mathbf{H}_{19} \mathbf{N}_{2} \mathbf{B r}\right)$. White powder, m.p. 124-126 ${ }^{\circ} \mathrm{C}$ (from THF). Found: C 55.09; H 6.65; N 9.71; Br 28.55. Calculated: C 55.13; H 6.76; N 9.89; $\mathrm{Br}$ 28.21\%. Yield 31\%. NMR data: $\delta_{\mathrm{H}} 9.91(\mathrm{H}-2), 8.15(\mathrm{H}-4), 7.7$ (H-5), $7.7(\mathrm{H}-6), 8.15(\mathrm{H}-7), 5.1$ $(\mathrm{CH}), 1.7\left(\mathrm{CH}_{3}\right) . \delta_{\mathrm{C}} 139.07(\mathrm{C}-2), 130.5(\mathrm{C}-8), 130.5$ (C-9), $126.4(\mathrm{C}-5), 126.4(\mathrm{C}-6), 114.02(\mathrm{C}-$ 4), $114.02(\mathrm{C}-7), 53.1(\mathrm{CH}), 28.2\left(\mathrm{CH}_{2}\right), 19.3\left(\mathrm{CH}_{3}\right)$.

1,3-di-iso-Butylbenzimidazolium bromide $\left(\mathbf{8}, \mathbf{C}_{15} \mathbf{H}_{23} \mathbf{N}_{2} \mathbf{B r}\right)$. White powder, m.p. $176-179^{\circ} \mathrm{C}$ (from THF). Found: C 57.87; H 7.64; N 8.85; Br 25.64. Calculated: C 57.88; H 7.45; N 9,00; Br 25.67\%. Yield 29\%. NMR data: $\delta_{\mathrm{H}} 9.9$ (H-2), 8.13 (H-4), 7.69 (H-5), 7.69 (H-6), $8.13(\mathrm{H}-7)$, $4.36\left(\mathrm{CH}_{2}\right), 2.24(\mathrm{CH}), 0.99\left(\mathrm{CH}_{3}\right) . \delta_{\mathrm{C}} 142.5(\mathrm{C}-2), 131.3(\mathrm{C}-8), 131.3(\mathrm{C}-9), 126.5(\mathrm{C}-5), 126.5$ (C-6), $113.9(\mathrm{C}-4), 113.9(\mathrm{C}-7), 53.1(\mathrm{CH}), 28.2\left(\mathrm{CH}_{2}\right), 19.3\left(\mathrm{CH}_{3}\right)$. 
General procedure for preparation of 1,3-dialkylimidazole-2-ilidenes and 1,3-dialkylbenzimidazole-2-ilidenes. All operations were carried out under dry argon. The solvents were purified using the methods described earlier, ${ }^{12}$ and deoxygenated immediately before use. Glassware was dried at $160^{\circ} \mathrm{C}$. To $0.1 \mathrm{mmol}$ of starting salt in $10 \mathrm{ml}$ of THF in a Schlenk tube was added $0.12 \mathrm{mmol}$ of sodium hydride (washed to remove oil). The mixture was stirred for 3 minutes, then $5 \mathrm{ml}$ of $5 \mathrm{mmol} \%$ potassium tert- butoxide THF solution was added under vigorous stirring. The mixture was stirred for $4 \mathrm{~h}$ and filtered to remove $\mathrm{NaBr}$. The filtrate was evaporated under vacuum to give the carbene.

1,3-di-iso-Propylimidazole-2-ilidene $\left(\mathbf{9}, \mathbf{C}_{\mathbf{9}} \mathbf{H}_{\mathbf{1 6}} \mathbf{N}_{\mathbf{2}}\right)$. The carbene was obtained in THF solution. NMR data: $\delta_{\mathrm{H}} 6.84(\mathrm{H}-4), 6.84(\mathrm{H}-5), 4.34(\mathrm{CH}), 1.35\left(\mathrm{CH}_{3}\right) . \delta_{\mathrm{C}} 205.39(\mathrm{C}-2), 115.41(\mathrm{C}-4)$, $115.41(\mathrm{C}-5), 51.44(\mathrm{CH}), 21.99\left(\mathrm{CH}_{3}\right)$.

1,3-di-iso-Butylimidazole-2-ilidene $\left(\mathbf{1 0}, \mathbf{C}_{\mathbf{1 1}} \mathbf{H}_{\mathbf{2 0}} \mathbf{N}_{2}\right)$. The carbene was obtained in THF solution. NMR data: $\delta_{\mathrm{H}} 6.96(\mathrm{H}-4), 6.96(\mathrm{H}-5), 3.88\left(\mathrm{CH}_{2}\right), 2.05(\mathrm{CH}), 0.84\left(\mathrm{CH}_{3}\right) . \delta_{\mathrm{C}} 202.68(\mathrm{C}-2)$, 119.39 (C-4), 119.39 (C-5), $57.36\left(\mathrm{CH}_{2}\right), 30.14(\mathrm{CH}), 19.10\left(\mathrm{CH}_{3}\right)$.

1,3-di-iso-Propylbenzilimidazole-2-ilidene $\left(\mathbf{1 1}, \mathbf{C}_{\mathbf{1 3}} \mathbf{H}_{\mathbf{1 8}} \mathbf{N}_{\mathbf{2}}\right)$. The carbene was obtained in THF solution. NMR data: $\delta_{\mathrm{C}} 221.6$ (C-2), 134.7 (C-8), 134.7 (C-9), 120.8 (C-5), 120.8 (C-6), 109.9 (C-4), $109.9(\mathrm{C}-7), 49.1(\mathrm{CH}), 23.1\left(\mathrm{CH}_{3}\right)$.

1,3-di-iso-Butylbenzimidazole-2-ilidene (12, $\left.\mathbf{C}_{\mathbf{1 5}} \mathbf{H}_{\mathbf{2} 2} \mathbf{N}_{\mathbf{2}}\right)$. The carbene was obtained in THF solution. NMR data: $\delta_{\mathrm{H}} 6.43(\mathrm{H}-4), 6.43(\mathrm{H}-5), 6.26(\mathrm{H}-6), 6.26(\mathrm{H}-7), 4.04(\mathrm{CH}), 2.161\left(\mathrm{CH}_{2}\right)$, $0.89\left(\mathrm{CH}_{3}\right) . \delta_{\mathrm{C}} 225.6(\mathrm{C}-2), 130.6(\mathrm{C}-8), 130.6(\mathrm{C}-9), 120.4(\mathrm{C}-5), 120.4(\mathrm{C}-6), 110.2(\mathrm{C}-4)$, $110.2(\mathrm{C}-7), 54.7(\mathrm{CH}), 29.3\left(\mathrm{CH}_{2}\right), 19.2\left(\mathrm{CH}_{3}\right)$.

\section{References}

1. Arduengo, III, A. J.; Harlow, R.L.; Kline, M. J. Am. Chem. Soc. 1991, 113, 361.

2. Bourissou, D.; Guerret, O.; Gabbaï, F. P.; Bertrand, G. Chem. Rev. 2000, 100, 39.

3. Herrmann, W.A. Angew. Chem. Int. Ed. 2002, 41, 1290.

4. Herrmann, W.A.; Elison, M.; Fischer, J.; Köcher, C.; Artus, G.R.J. Chem. Eur. J. 1996, 2, 772.

5. Herrmann, W.A.; Köcher, C.; Gooßen, L.J.; Artus, G.R.J. Chem. Eur. J. 1996, 2, 1627.

6. Jafarpour, L.; Stevens, E.D.; Nolan, S.P. J. Organomet. Chem. 2000, 606, 49.

7. Kuhn, N.; Kratz, T. Synthesis 1993, 6, 561.

8. Arduengo, III, A.J.; Dias, H.V.R.; Harlow R.L.; Kline, M. J. Am. Chem. Soc. 1992, 114, 5530 .

9. Olofson, R.A.; Kendall, R.V. J. Org. Chem. 1970, 35, 2246.

10. Tarasova, O.A.; Shmidt, E.Yu.; Baikalova, L.V.; Mikhaleva, A.I.; Trofimov, B.A. Izv. Akad. Nauk Ser. Khim. 1997, 11, 2005.

11. Seoud, O.; Aldrigue, W. Anaic. de Academia Brass. Ciens. 1978, $50,87$.

12. Bogdal, D.; Pielichowski, J.; Jaskot, K. Heterocycles 1997, 45, 715.

13. Herrmann, W.A.; Elison, M.; Fischer, J.; Köcher, C. Chem. Eur. J. 1996, 2, 772.

14. Gstöttmayr, C.W.K.; Böhm, V.P.W.; Hardwick, E.; Grosche, M.; Herrmann, W.A. Angew. Chem. Int. Ed. 2002, 41, 1363. 